\title{
ESTABLISHMENT AND UTILITY OF TRANSPLANTABLE OSTEOSARCOMAS IN FISHER 344 RATS
}

\author{
Tatsusuke Sato \\ Department of Medical Technology, School of Allied Medical Sciences, Hirosaki University
}

\begin{abstract}
Two subcutaneous transplantable rat osteosarcoma lines were established serially and used to analyze the mechanism of radiographic sclerotic changes in human osteogenic sarcomas following chemotherapy. Primary osteogenic sarcomas were induced in the left humerus (POA) and in the right femur (POB) by [ $\left.{ }^{32} \mathrm{P}\right] \mathrm{H}_{3} \mathrm{PO}_{4}$ in one F344 male rat. Pieces of POA or POB tumors measuring about 1 $\mathrm{mm}^{3}$ were transplanted subcutaneously into syngeneic male rats up to the 22nd generation, for a period of three years and six months. Both the POA and POB lines maintained osteoid and bone formation, and alkaline phosphatase (ALP) activity. Moreover, tumor nodules grown afler transplantation of the POA or POB line showed severe radiographic sclerotic changes three or four weeks after a single dose of cis-diamminedichloroplatinum (CDDP). Histologically, the sclerotic changes were caused by increased bone formation. (J Toxicol Pathol 3: 39 44, 1990)

Key words: Transplantable osteosarcoma, Bone formation, Chemotherapy, Radiographic sclerotic change, F344 rat
\end{abstract}

\section{Introduction}

An animal model of a human disease is useful for studying that disease, and the histological features are considered to be similar to those in humans. Primary osteogenic sarcomas in laboratory animals histologically resemble human osteogenic sarcomas with respect to bone or osteoid formation and alkaline phosphatase (ALP) activity, but this bone or osteoid formation usually disappeares after serial transplantation ${ }^{1-3}$, except for a report by Mii et al 4 .

Recently I established two subcutaneous transplantable osteosarcoma cell lines producing bone and osteoid tissue in Fisher 344 rats. These two cell lines still retain ALP activity, and moreover have been found to be useful for analyzing the mechanism of radiographic sclerotic changes in

\section{佐藤達瓷}

Accepted for publication : April 14, 1990

Mailing address: Tatsusuke Sato, Department of Medical Technology, School of Allied Medical Sciences, Hirosaki University, 66-1 Honcho, Hirosaki 036, Japan. human osteogenic sarcomas following intensive chemotherapy ${ }^{5}$.

\section{Materials and Methods}

1. Primary tumors and syngeneic transplantation

Primary osteogenic sarcomas were induced in a rat using a radioisotope $\left(\left[{ }^{32} \mathrm{P}\right] \mathrm{H}_{8} \mathrm{PO}_{4}\right)$. A male rat (Fisher F344/Du Crj) was intraperitoneally administered $0.5-1.0 \mu \mathrm{Ci} / \mathrm{g}$ body wt. of the isotope once every two or three weeks. The total number of doses of [ $\left.{ }^{32} \mathrm{P}\right] \mathrm{H}_{3} \mathrm{PO}_{4}$ was 13 , up to $1722 \mu \mathrm{Ci}$. Two primary tumors were induced in the same rat about six months after the final dose of $\left[{ }^{32} \mathrm{P}\right] \mathrm{H}_{3}$ $\mathrm{PO}_{4}$. One tumor produced in the left humerus was named POA and the other in the right femur was named POB.

About $1 \mathrm{~mm}^{3}$ of POA or POB was transplanted into the subcutaneous tissue of each of four male syngeneic rats. The first transplantations were termed POA 1 or POB 1 . These transplantations were repeated for 22 generations using four male syngeneic rats once every month or three months. 


\section{Search for bone or asteoid formation}

Bone formation in the tumor tissue was investigated by radiological examination with a soft $X$-ray apparatus (Softex type EMB, Softex Co., Tokyo) and histological examination with hematoxylin and eosin (HE) staining and von Kossa's silver method for calcium.

Osteoid formation in the tumor tissue was examined histologically by Pierce's method ${ }^{8,7}$ using Technovit (Kulzer Co., West Germany)-embedded sections.

\section{Search for ALP activity}

For light microscopic observation, ALP activity in tumor tissue was investigated by the azo-dye method for ALP on frozen sections. For electron microscopic observation, the localization of ALP activity in POA 10, POA22, and POB9, POB22 was investigated by the lead citrate method ${ }^{\mathrm{a}}$.
4. Search for radiographic sclerotic changes after chemotherapy

Five male F344 rats received transplants of the POA or POB line subcutaneously. When the tumor size reached about $4 \times 3 \times 3 \mathrm{~cm}$, a single dose of $5 \mathrm{mg} / \mathrm{kg}$ cis-diamminedichloroplatium (CDDP) was injected i.v. into five tumor-bearing rats. Two, 3 and 4 weeks after the administration of CDDP, radiographic sclerotic changes were examined.

\section{Results}

\section{Primary tumors}

Radiographic examination of a rat injected with [ $\left.{ }^{32} \mathrm{P}\right] \mathrm{H}_{3} \mathrm{PO}_{4}$ into the peritoneal cavity showed two primary tumors in the left humerus (POA) and the right femur (POB) (Fig. 1). POA and POB both demonstrated bone formation.

Histologically, POA showed net-like bone formation (Fig. 2). and fibroblastic tumor cells

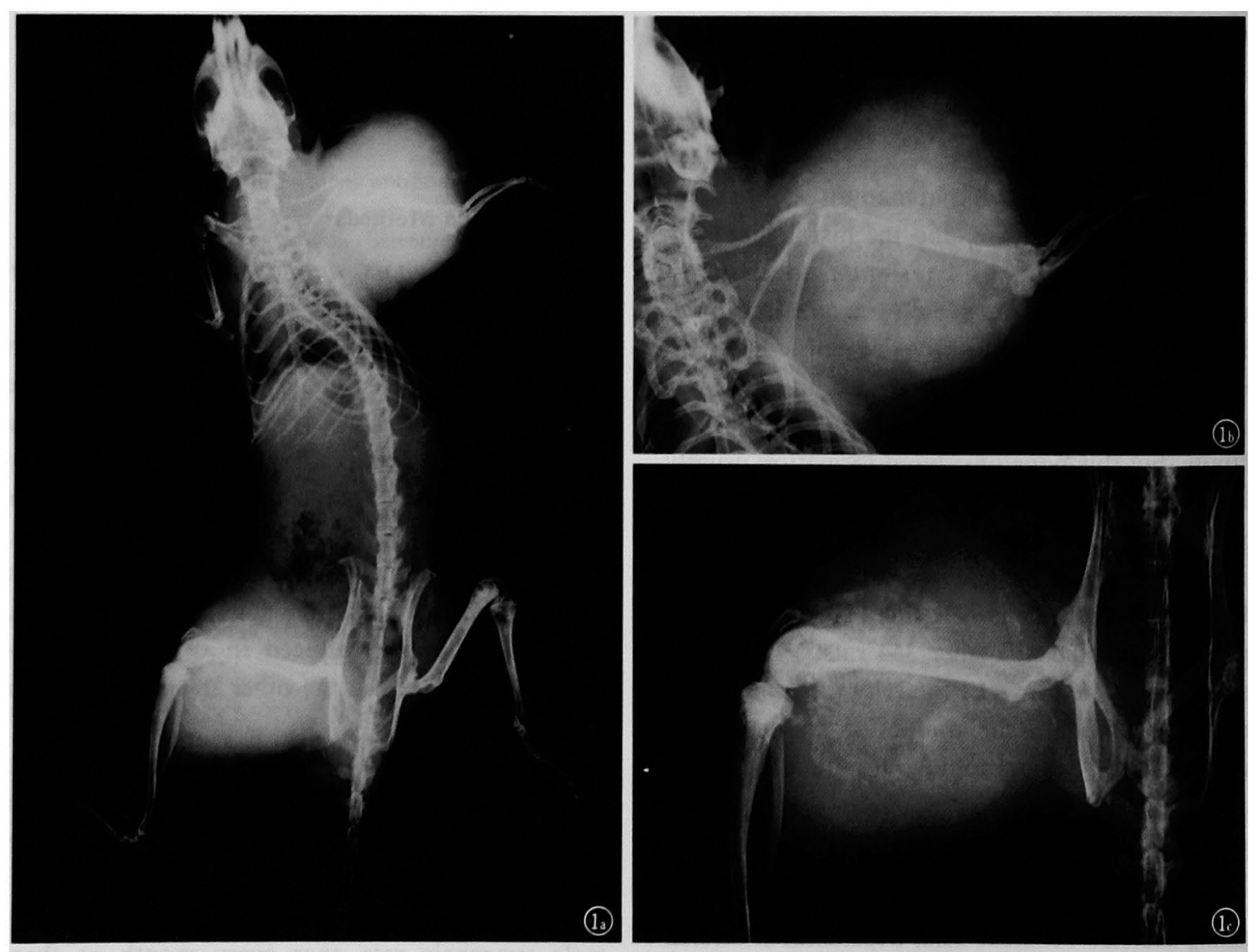

Fig. 1. Radiographical features of the primary tumors in the rat; (a) tumors of the left humerus (POA) and right femur (POB), (b) POA, and (c) POB demonstrate bone formation. 

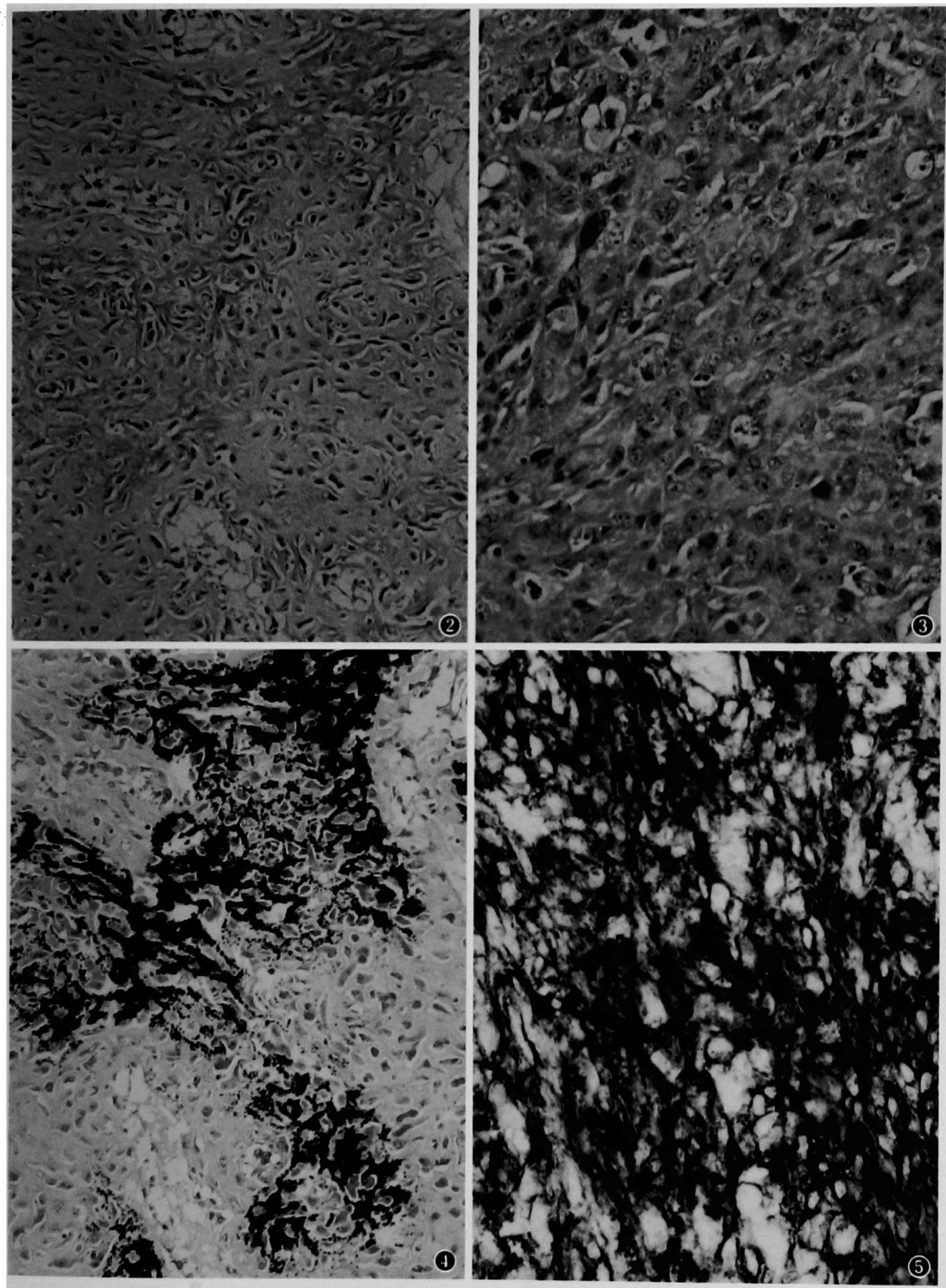

Fig. 2. Net-like bone and osteoid formation in POA. (HE, original magnification $\times 150$ ).

Fig. 3. Moderate nuclear pleomorphism in POA (HE, original magnification $\times 300$ ).

Fig. 4. Calcified bone formation in POA (von Kossa's silver method, original magnification $\times 150$ ).

Fig. 5. Strongly positive reaction for ALP reaction in POA. (azo-dye method, original magnification $\times 300$ ). 
with moderate nuclear pleomorphism (Fig. 3) were evident upon HE staining. von Kossa's silver method demonstrated calcified bone formation in POA (Fig. 4). Pierce's method revealed redstaining net-like osteoid formation in POA. From the above findings, POA was diagnosed as osteosarcoma. ALP staining showed strong ALP activity on the tumor celis (Fig 5).

The histological features of POB were exactly the same as those of POA except for mild nuclear pleomorphism. POB was also diagnosed as osteosarcoma.

No metastasis of POA or POB to any organ was noted.

2. Course of establishment of transplantable osteasarcoma cell lines

After two weeks, the syngeneic grafts (POA I and $P O B$ 1) of the first transplant generation had grown into palpable nodules. After one month, each of the four nodules of POA 1 and POB 1 had grown to thumb-tip size. The transplantation rate for both POA $I$ and POB 1 was 100\%, and the rates for generations 2 to 22 of the POA line were 50-100\% (mean 94.3\%). The transplantation rates for POB between generations 2-22 were 75-100\% (mean 97.7\%).

Metastasis of transplanted tumors to the bilataral lungs was recognized in only one rat with POB 7.

The total number of transplant generations for both the POA and POB lines was 22 generations, and the total transplantation period for the two lines of POA and POB has now reached three years and six months.

3. Relation between transplant generation and formation of bone or osteoid

The grade of bone and osteoid formation from POA 1 to POA 22, from POB 1 to POB 8 and from

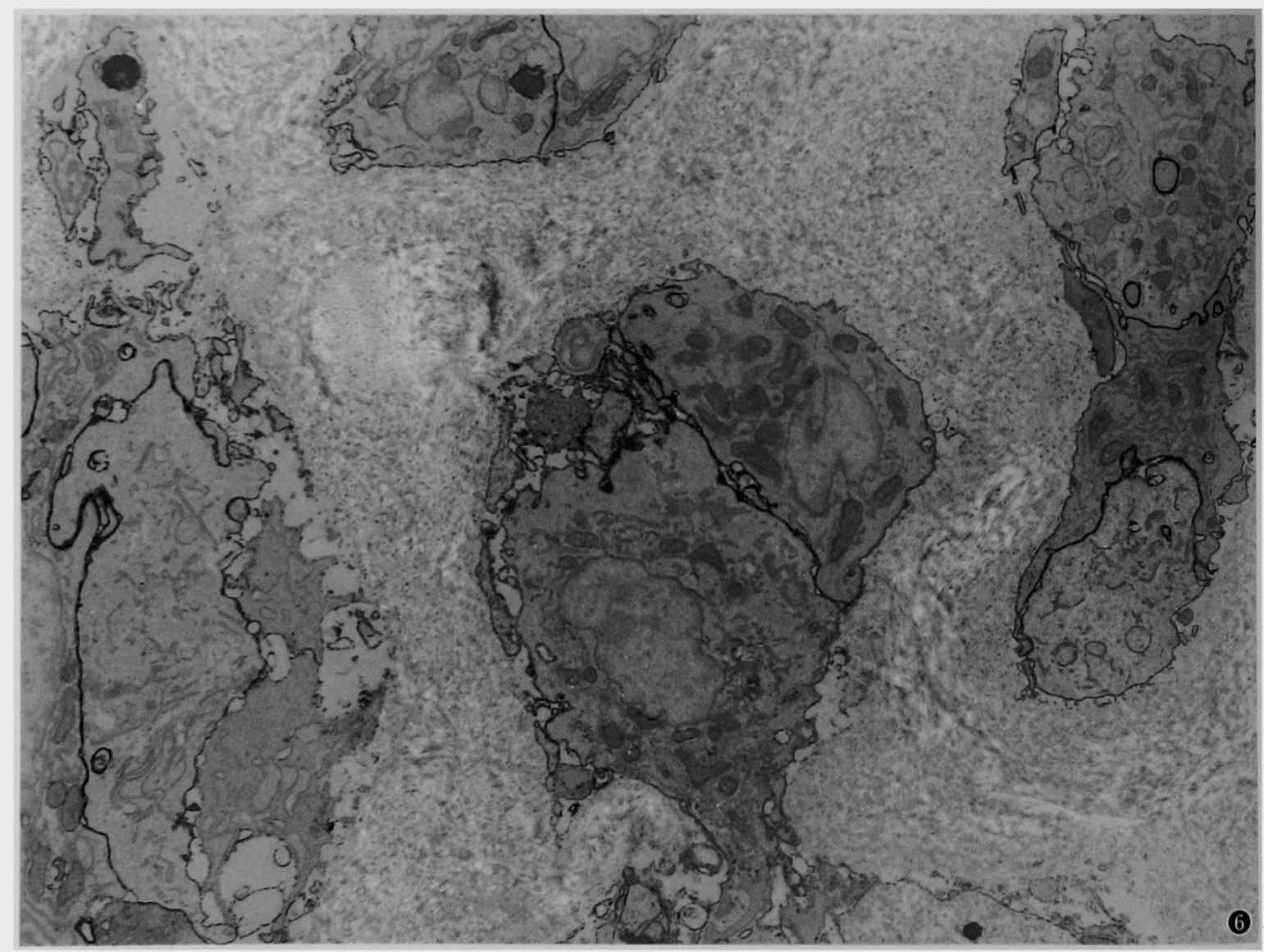

Fig. 6. Electron microscopic ALP activity in osteosarcoma cell lines. Note the marked product on the plasma membrane of POA 22 (lead citrate method, original magnification $\times 4,030$ ). 


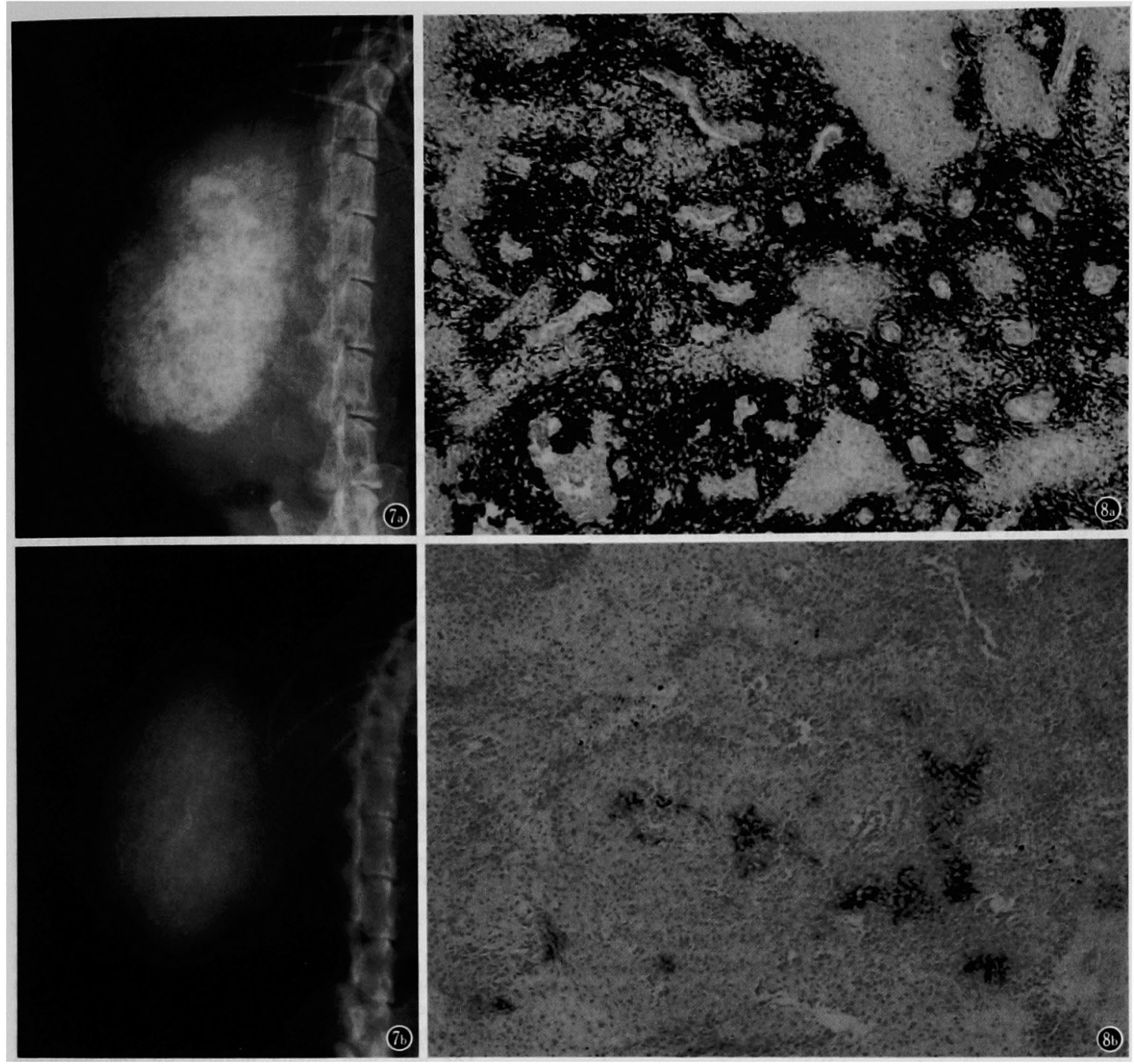

Fig. 7. Radiographic sclerotic changes in tumor nodules; (a) severe sclerotic change in POA four weeks after chemotherapy ; (b) mild sclerotic figures in POA without chemotherapy.

Fig. 8. Calcified bone formation in tumor nodules: (a) markedly calcified bone formation in POA four weeks after chemotherapy ; (b) mildly calcified bone formation in POA without chemotherapy (von Kossa's silver method, original magnification $\times 60$ ).

POB 12 to POB 22 was the same as that in the primary tumors POA and POB. The degree of bone and osteoid formation was reduced from POB 9 to POB 11, and many parts of these tumors showed a fibroblastic pattern.

4. Relation between transplant generation and ALP activity

ALP activity in tumor cells of all the transplant generations was strongly positive, as for the primary tumors POA and POB.

Electron microscopically, reaction product indicating ALP activity was recognized on the surface membrane of the tumor cells (Fig. 6).

5. Radiographic sclerotic changes after chemotherapy

By soft X-ray examination, tumor nodules that grew following transplantation of POA 22 and POB 22 showed medium sclerotic changes after two weeks in comparison with the situation before CDDP injection. Three and four weeks after chemotherapy, the sclerotic changes were severe and resembled those in human osteosarcoma after 
Table 1. Increase of Radiographic Sclerotic Change Four Weeks after Chemotherapy

\begin{tabular}{ccc}
\hline \multirow{2}{*}{$\begin{array}{c}\text { Rat Osteosarcoma } \\
\text { Line }\end{array}$} & \multicolumn{2}{c}{ CDDPa) } \\
\cline { 2 - 3 } & $+^{\mathrm{b})}$ & $-\mathrm{c}$ \\
\hline POA & $5 / 5$ & $0 / 5$ \\
POB & $4 / 5$ & $0 / 5$ \\
\hline
\end{tabular}

a) : cis-diaminedichloroplatinum

b) : Groups given CDDP injection

c) : Groups without CDDP injection

chemotherapy (Fig. 7 ). The results regarding the increase in radiographic sclerosis are recorded in Table 1. Five out of five animals in the POA group, and four out of five animals in the POB group injected with CDDP showed an increase in sclerotic figures.

By histological examination using von Kossa's method, the increased radiographic sclerotic changes in the groups given chemotherapy were due to increased bone formation (Fig. 8).

\section{Discussion}

Although bone or osteoid formation and ALP activity are recognized in primary osteosarcoma in laboratory animals, bone or osteoid formation usually disappears during serial transplantation ${ }^{1-3}$. Recently Mii et al.4 reported the establishment of transplantable cell lines of rat osteosarcoma producing osteoid and bone tissues. In this study I also succeeded in establishing subcutaneously transplantable osteosarcoma cell lines producing bone and osteoid tissue, and showing ALP activity.

It is reported that human osteosarcomas after chemotherapy show radiographic sclerotic changes $^{5}$ and a histological increase in tumorous osteoid $^{9.10}$. The mechanism of appearance of these sclerotic changes is presumed to involve normalization of tumor cells ${ }^{9}$ or bone morphogenetic protein (BMP) ${ }^{11}$. Smith $e t a l^{5}$. proposed that the dramatic sclerotic changes after chemotherapy were the result of destruction of tumor cells, followed by attempted healing through reactive bone formation. However, although their hypothesis may not explain all of their findings, they feel that it is a useful model which well matches their observations. Using the present osteosarcoma cell lines established here, it was proved that the severe radiographic sclerotic changes after injection of CDDP were caused by increased bone formation. Therefore, these cell lines should prove to be useful models for analysis of the mechanism of radiographic sclerotic changes in human osteogenic sarcomas following intensive chemotherapy.

\section{References}

1. Maibenco, HC, Krehbiel, RH, and Nelson, D: Transplantable osteogenic tumor in the rat. Cancer Res 27 : 362-366, 1967.

2. Albala, $M M$ and Esparza, AR: Transplantable osteogenic sarcoma in inbred AKR mice. Cancer Res 29 : 1517-1522, 1969.

3. Nakakuki, K, Shimokawa, K, Yamauchi, H, and Ojima, A : A spontaneous transplantable osteogenic sarcoma in AKR/Ms mice. Gann 67: 513-521, 1976.

4. Mii, Y, Tsutsumi, M, Shiraiwa, K, Miyauchi, $Y$, Hohnoki, K, Maruyama, H, Ogushi, H, Masuhara, K, and Konishi, $Y$ : Transplantable osteosarcomas with high lung metastatic potential in Fischer 344 rats. Jpn J Cancer Res (Gann) $79: 589-592,1988$.

5. Smith. J, Heelan, RT, Huvos, AG, Caparros, B, Rosen, G, Urmacher, C, and Caravelli, JF : Radiographic changes in primary osteogenic sarcoma following intensive chemotherapy. Radiology 143: 355-360, 1982.

6. Pierce. $M$ : Connective tissue staining in water soluble methacrylate. Histo-Logic 16 : 216-217, 198586.

7. Sato. T, Nakano, K, and Kakizaki, H : Utility of Pierce's method for differentiation of osteoid in osteosarcoma from connective tissue hyaline(in Japanese). Pathol Clin Med 6 : 1217-1220, 1988.

8. Mayahara. H, Hirano, H, Saito, T, and Ogawa, K : The new lead citrate method for the ultracytochemical demonstration of activity of non-specific alkaline phosphatase (orthophosphoric monoester phosphohydrolase). Histochemie 11: 88-96, 1967.

9. Marroum, M-C, Huvos, AG, and Rosen, G: Pathologic aspects of chemotherapy response in the treatment of osteogenic sarcoma: an analysis of two cases. Oncology 34: 273-280, 1977.

10. Tateishi, A, Takeyama, S, Higaki, S, Tanaka, H, and Yamanashi, $M$ : Preoperative aggresive chemotherapy including isolation perfusion as an adjuvant of limb salvage operation for osteosarcoma. Limb Salvage in Musculoskeletal Oncology, pp. 286-294, Churchill Livingstone, New York/Edinburgh/London/Melbourne, 1987.

11. Takaoka, K, Yoshikawa, H, Shimizu, N, Ono, K, Amitani, K, Nakata, $Y$, and Sakamoto, $Y$ : Purification of a bone-inducing substance (osteogenic factor) from a murine osteosarcoma. Biomed Res 2: 466-471, 1981. 